\title{
A realidade da saúde bucal do idoso no Sudeste brasileiro
}

\author{
The reality of the oral/dental health of the elderly \\ in the Brazilian Southeast
}

Ana Carolina de Assis Simões ${ }^{1}$

Denise Maciel Carvalho ${ }^{2}$

${ }^{1}$ Universidade Federal do Triângulo Mineiro. Av. Frei Paulino 30, Abadia. 38025-180 Uberaba MG. aninhassim@yahoo.com.br ${ }^{2}$ Secretaria de Estado de Saúde de Minas Gerais.
Abstract The scope of this work was to describe the alterations revealed in oral/dental structures resulting from ageing and to verify, through the national scientific production, with emphasis on the Southeastern region, the prevaling oral/dental problems in the elderly. A review of the literature on the theme of ageing in the context of dentistry was conducted. The results pointed to the fact that the elderly population is the fastest growing population segment. The prevailing oral/dental problems in this age bracket are radicular caries and periodontal illness, which contribute to the vast majority of dental extractions. In the Southeastern region of Brazil, the use of prostheses is low when compared with the high rate of tooth loss and the percentage of partially toothed elderly people; the value of the CPOD index is high and the elderly have a positive perception on their teeth, despite the fact that oral/dental problems have a negative impact on their lives. It is concluded that the inclusion of a dental surgeon in the healthcare team of the institutions and the systematization of oral/dental hygiene are pressing needs, in addition to the need for ongoing work of guidance for the the oral/dental healthcare of the elderly. Broadening of the study in this area is also fundamental to ensure dental care and treatment of these individuals.

Key words Ageing, Dentistry, Elderly, Oral/dental health
Resumo Este trabalho teve como objetivos descrever as alterações manifestadas nas estruturas bucais decorrentes do envelhecimento e verificar, através da produção científica nacional, com ênfase na Região Sudeste, os problemas bucais prevalentes nos idosos. Foi realizada uma revisão de literatura abordando a temática envelhecimento no contexto da odontologia. Os resultados apontaram que a população idosa é o segmento populacional que mais cresce. Os problemas bucais prevalentes nessa faixa etária são as cáries radiculares e a doença periodontal, que contribuem para a grande maioria das extrações dentárias. $\mathrm{Na}$ Região Sudeste do Brasil, o uso de prótese é baixo quando comparado à alta taxa de edentulismo $e$ ao percentual de idosos parcialmente dentados; o valor do indice CPOD é alto e os idosos apresentam uma percepção positiva sobre seus dentes, apesar de os problemas bucais terem impacto negativo nas suas vidas. Conclui-se que a incorporação do cirurgião-dentista na equipe de saúde das instituições e a sistematização das práticas de higiene bucal são necessidades reais, além da necessidade de um trabalho continuado de orientação para o cuidado da saúde bucal do idoso. É também fundamental a ampliação do estudo nessa área, visando qualificar a atenção e o tratamento odontológico para esses indivíduos.

Palavras-chave Envelhecimento, Odontologia, Idoso, Saúde bucal 


\section{Introdução}

Ao longo dos anos, acompanhamos uma evolução dos conceitos de saúde e bem-estar, de prevenção de doenças e promoção da saúde, além de um despertar para maior conscientização da importância dos cuidados com a boca e com os dentes em todas as fases da vida. Sabe-se que o Brasil, semelhante aos diversos países do mundo, está envelhecendo rapidamente. A população idosa, composta por pessoas com mais de 60 anos de idade, compõe atualmente o segmento populacional que mais cresce em termos proporcionais ${ }^{1}$.

Os problemas de saúde modificam-se com o decorrer dos anos. O envelhecimento leva a várias alterações fisiológicas em todo o organismo, havendo uma grande prevalência de doenças crônicas nesses indivíduos, que constituem a maior parcela de pessoas que necessitam de atendimento nos serviços de saúde ${ }^{2}$.

A odontologia reuniu um corpo de doutrina importante relativo aos efeitos do tempo sobre a boca, dentes e estruturas anexas. Nasceu assim a odontogeriatria, que deve ser vista com seriedade, acima dos interesses pessoais dos envolvidos, visando ao bem-estar do idoso, um ser geralmente oprimido por aposentadorias escorchantes e vivendo em cidades não preparadas para acolhê-los, principalmente em países latinos como o Brasil ${ }^{3}$.

A saúde bucal na terceira idade é um fator indispensável para o envelhecimento saudável e uma boa qualidade de vida, mas sua importância ainda não é devidamente reconhecida. Segundo Pucca Júnior ${ }^{4}$, o quadro de saúde bucal da terceira idade reflete nitidamente as condições desiguais em que as pessoas vivem e trabalham.

A perda total dos dentes (edentulismo) ainda é aceita por muitos como um fenômeno natural do envelhecimento. No entanto, sabe-se hoje que esse fato é o reflexo da falta de prevenção, de informação e consequentemente de cuidados com a higiene bucal, que deveriam ser destinados principalmente à população adulta, para que mantenha seus dentes até idades mais avançadas.

A prevalência de doença periodontal aumenta com o decorrer da idade e tem sido vista, por alguns autores, como a principal causa do edentulismo, resultando num grande número de pessoas necessitando de próteses ${ }^{1}$. Outra razão para a perda dental é a evolução lenta de cáries radiculares, infiltrações e cáries sob restaurações e próteses, assim como fraturas que normalmente ocorrem pela má distribuição das forças mastigatória ${ }^{6}$.
Pucca Júnior $^{4}$ explica que esse quadro precário é decorrente não só do processo de envelhecimento por si, mas principalmente de um conjunto de agravos diferenciados que atingem os idosos de maneiras distintas durante a vida. Segundo o autor, os recursos básicos para orientação do idoso envolvem informações quanto à limpeza regular diária dos dentes e próteses, controle da dieta e orientações visando ao fortalecimento da superfície dentária, principalmente com o uso do flúor.

Nesse contexto, conhecer a realidade da saúde bucal do idoso e alguns de seus fatores determinantes constitui-se na motivação para este estudo, que pretende apresentar uma visão do envelhecimento e das modificações da saúde bucal em razão do decorrer da idade, assim como enfatizar a necessidade de estratégias de educação em saúde para um envelhecimento saudável.

Este estudo tem como objetivos descrever as alterações manifestadas nas estruturas bucais decorrentes do envelhecimento e verificar, através da produção científica nacional, com ênfase na Região Sudeste, os problemas bucais prevalentes nos idosos.

\section{Material e método}

Para o presente trabalho, optou-se por uma revisão de literatura. A fim de atender aos objetivos propostos, foi realizado um levantamento bibliográfico no acervo da Biblioteca da Universidade de Uberaba e da Universidade Federal de Minas Gerais, além de publicações on-line.

Para selecionar os artigos, foram utilizadas as seguintes palavras-chaves: saúde bucal, idoso, envelhecimento, epidemiologia, odontogeriatria. Ressalta-se que não foi limitado um período específico de publicação, seguindo-se apenas o critério de inclusão estabelecido, ou seja, abordar a temática envelhecimento no contexto da odontologia.

Após o levantamento do material, as seguintes etapas foram percorridas: organização, leitura exploratória e reconhecimento dos artigos que interessavam à pesquisa, através da qual foi possível conhecer o conteúdo específico; seleção das referências que atendiam aos propósitos da pesquisa; leitura e análise dos textos selecionados, destacando-se os principais aspectos abordados sobre o tema. Posteriormente, procedeu-se à síntese e à interpretação dos textos, bem como à organização das informações para a apresentação. 


\section{Apresentação e discussão dos resultados}

\section{O envelhecimento e os aspectos bucais}

Geralmente, os pacientes geriátricos apresentam um quadro clínico comum. O cuidado geriátrico inclui pelo menos diagnóstico, prevenção e tratamento da cárie, doenças periodontais, dores de cabeça e pescoço, disfunções salivares, problemas com a prótese e comprometimento das funções de mastigação, deglutição e paladar ${ }^{7}$.

As estruturas bucais sofrem ação do tempo, e várias são as adaptações fisiológicas que se processam durante o ciclo da dentição normal. A dentina depositada continuamente diminui o tamanho da câmara pulpar, provocando a atresia dos condutos radiculares. Os tecidos periodontais sofrem retração, perda do colágeno e ficam mais suscetíveis a inflamação. As alterações mastigatórias são inúmeras, decorrentes da perda dos elementos dentais, uso de próteses, restaurações realizadas ao longo da vida e hábitos alimentares e funcionais ${ }^{6}$.

Com o aumento da população de idosos, é necessário ter uma demanda crescente e diversificada para a atenção e para o tratamento odontológico desses indivíduos.

Araújo et $a l .{ }^{8}$, revisando a literatura, dissertaram sobre o crescente envelhecimento da população mundial, inclusive no Brasil, ressaltando a importância de medidas para se lidar com tal situação. Segundo a pesquisa, no Brasil a maioria dos idosos é do sexo feminino; vive em domicílios multigeracionais; é referência econômica neles; possui baixo nível socioeconômico; é portadora de, pelo menos, uma doença crônica; independente para realização das atividades da vida diária; não possui dentes, e busca atenção em saúde no Sistema Único de Saúde (SUS). Os idosos brasileiros expostos a situações de vulnerabilidade social estão sujeitos à interferência direta dos determinantes sociais no processo saúde-doença. O Suporte Social inclui políticas e redes de apoio social, que atuam como agente de integração do idoso na sociedade, minimizando os riscos de exclusão social e, conseqüentemente, de danos à sua saúde por meio de medidas de Promoção de Saúde. O estudo aborda o Suporte Social e alguns de seus aspectos, tais como: tipo e local de residência, transporte e suporte financeiro; idosos brasileiros e sua relação com a Promoção de Saúde.

Em estudo realizado por Cormack ${ }^{1}$, buscouse uma interação das questões relativas à odontologia ao contexto dos estudos geriátricos e ge- rontológicos, em que o processo do envelhecimento se relacione com a manutenção da saúde oral. Através de uma revisão da literatura foram apresentados alguns dados relativos ao crescimento da população idosa e à mudança do perfil epidemiológico do "novo idoso" que surge, com diferentes necessidades orais em relação à geração anterior, tais como a diminuição do edentulismo, o aumento das cáries das superfícies radiculares e o incremento da doença periodontal. Concluiu-se que, com o aumento da população idosa, teremos nos próximos anos uma demanda por maior e mais diversificada atenção odontológica para esse grupo em particular. A profissão odontológica - incluindo associações de classe, o meio universitário e os diversos prestadores de serviço - deve estar ciente e alerta para essa questão, de forma a ampliar o estudo e a pesquisa nessa área.

Brunetti et al. ${ }^{3}$, aprimorando os estudos do envelhecimento, diante de uma revisão de literatura constataram aumento significativo no número de pacientes de terceira idade. Observaram existir um número de profissionais insuficientes e com pouco preparo para o atendimento desses indivíduos em nosso país, realçando a necessidade de profundos estudos e adequação técnica para atuarem nessa população. Concluíram que a odontologia deverá dedicar-se cada vez mais ao atendimento de idosos, considerando suas maiores perspectivas de vida, exigindo grande motivação no estudo das particularidades dessa faixa etária.

Carneiro et al. ${ }^{9}$, em levantamento epidemiológico realizado em São Paulo (SP), utilizando dois grupos de idosos com 60 anos ou mais (examinados em domicílio e em instituições beneficentes), mostram que a população de terceira idade apresenta condições muito precárias de saúde bucal. O índice CPOD variou entre 23,0 e 31,0 , sendo que aproximadamente $75 \%$ dos idosos eram edêntulos e apenas 50\% portadores de prótese total. Bolsas periodontais predominaram em relação às demais patologias em $65 \%$ dos idosos. Esse quadro demonstra que a cárie e a doença periodontal contribuem para mais de 90\% das extrações dentárias.

Meneghim et al. ${ }^{10}$ verificaram as condições periodontais e lesões radiculares de uma amostra de população idosa, institucionalizada, do município de Piracicaba (SP). Nesse estudo, foram examinados 151 idosos com idade entre 54 e 93 anos. Os sextantes foram examinados e classificados do ponto de vista de saúde periodontal, necessidade de tratamento periodontal, presença de re- 
tração gengival e lesões radiculares, utilizando-se como critérios os indicadores da Organização Mundial da Saúde (OMS). Comparando-se duas faixas etárias, de pacientes com idade entre 50 e 75 anos e pacientes com idade acima de 75 anos, constatou-se que há aumento na prevalência de lesões de cárie e outras afecções radiculares, como erosão e abrasão, indicando aumento na suscetibilidade a tais lesões com o passar da idade.

Silva e Valsecki Júnor ${ }^{11}$ avaliaram as condições de saúde bucal em pessoas com 60 anos ou mais no município de Araraquara, estado de São Paulo, no ano de 1998. Participaram do estudo 194 pessoas (91 institucionalizadas, com idade média de 73,6 anos, e 103 não institucionalizadas, com idade média de 69,3 anos), que foram examinadas por um cirurgião-dentista, previamente treinado, para determinar a prevalência das principais doenças bucais. Os resultados mostraram um grande número de pessoas edentadas $(72 \%$ dos institucionalizados e $60 \%$ dos não institucionalizados) e de dentes extraídos (93 e 90\%, respectivamente), grande frequência de bolsas periodontais (57 e 75\%) e de próteses inadequadas ( $80 \%$ das próteses em pessoas institucionalizadas e $61 \%$ em pessoas não institucionalizadas). Os resultados mostram o comprometimento da qualidade de vida de grande parte dessas pessoas e indicam a necessidade de maior atenção dos serviços públicos a esse grupo populacional.

Colussi e Freitas ${ }^{5}$ fizeram uma revisão crítica dos estudos epidemiológicos apresentados nas publicações nacionais, a partir de 1988, com relação à saúde bucal do idoso. Foram encontrados 29 artigos, dos quais sete estudos foram analisados quanto à metodologia utilizada (faixa etária, tipo de amostra, forma de apresentação dos dados) e quanto aos resultados apresentados (índice CPOD, participação de dentes extraídos, percentual de edêntulos e, uso e necessidade de prótese). O CPOD encontrado variou de 26,8 a 31,0 , sendo que o componente extraído representou cerca de $84 \%$ desse índice. A prevalência do edentulismo ficou em $68 \%$. Somente $3,9 \%$, um pequeno percentual dos idosos, não necessitava nem usava qualquer tipo de prótese, e o uso da prótese total foi mais frequente no arco superior do que no inferior. A falta de padronização na organização e na apresentação dos dados, assim como a sua escassez, prejudicou a análise, sugerindo que deva haver maior preocupação por parte dos pesquisadores quanto à realização de novas pesquisas e, principalmente, quanto à maneira como estas serão conduzidas. Apesar disso, confirmaram-se as condições muito precárias de saúde bucal do Brasil.
Moreira et al. ${ }^{12}$ objetivaram realizar uma revisão sistemática da literatura, no período de 1986 a 2004, sobre os problemas bucais mais prevalentes entre os idosos brasileiros e conhecer os principais obstáculos no acesso aos serviços de saúde. Os valores médios do índice CPOD encontrados nos artigos pesquisados foram de 25,0 a 31,0. Verificou-se grande porcentagem de indivíduos edêntulos. As principais barreiras quanto ao acesso aos serviços odontológicos foram a baixa escolaridade, a baixa renda e a escassa oferta de serviços públicos de atenção à saúde bucal. Diante desses fatores, a saúde bucal do idoso brasileiro encontra-se em situação precária, com elevados índices de edentulismo, refletindo a ineficácia historicamente presente nos serviços públicos de atenção odontológica, limitando a extrações em série e serviços de urgência, com base no modelo assistencial privatista ${ }^{12}$.

Ferreira et al. ${ }^{13}$ avaliaram a condição de saúde bucal dos idosos residentes em instituições de longa permanência de Belo Horizonte (MG). Participaram do estudo 335 indivíduos com 60 anos de idade ou mais selecionados por amostragem aleatória, estratificada e proporcional. Os dados foram coletados por meio de pesquisa nos registros da instituição, entrevistas com os idosos e exame clínico da cavidade bucal por um único pesquisador. A saúde bucal foi avaliada quanto a ocorrência de alterações de normalidade e lesões de mucosa bucal, edentulismo, uso de próteses, higiene bucal, experiência de cárie coronária e radicular e doença periodontal. Uma ou mais lesões ou alterações de normalidade da mucosa bucal foram observadas em 79,7\% dos idosos. As alterações de normalidade mais frequentemente observadas foram varicosidades sublinguais $(51,6 \%)$ e língua saburrosa $(27,8 \%)$. Estomatite por dentadura foi a lesão relacionada ao uso de prótese mais frequente $(15,2 \%)$. Edentulismo foi observado em $74,9 \%$ dos idosos, e destes, $42,6 \%$ não usavam prótese. Entre os parcialmente dentados $(25,1 \%), 30,9 \%$ usavam prótese parcial removível. Depósitos de placa bacteriana estavam presentes em $76,0 \%$ das superfícies dentárias avaliadas e $57,0 \%$ dos idosos usuários de prótese total superior apresentaram placa em pelo menos uma das superfícies dela. $\mathrm{O}$ índice CPOD foi de 30,8 (DP=3,2), com o componente perdido representando $94,2 \%$. Das 584 raízes expostas na cavidade bucal, 76,0\% encontravam-se hígidas, $14,4 \%$ cariadas e $9,6 \%$ restauradas. A grande maioria dos idosos $(78,2 \%)$ apresentou como pior condição periodontal sextante nulo e, entre os sextantes válidos, houve 
predomínio de bolsa periodontal de 4 a $5 \mathrm{~mm}$ $(\mathrm{IPC}=3)$. Os resultados mostram alta prevalência de alterações de normalidade e lesões de mucosa bucal entre os idosos. O uso de prótese é baixo, quando comparado à alta taxa de edentulismo e ao percentual de idosos parcialmente dentados. A higiene bucal dos idosos institucionalizados, em geral, é precária. Um alto valor de CPOD revela a severidade da doença cárie e suas consequências entre os idosos. Entre os dentes remanescentes, a experiência de cárie radicular é baixa. A doença periodontal acomete os poucos dentes remanescentes, que apresentam prioritariamente bolsas periodontais rasas.

Rodrigues et al. ${ }^{14}$ realizaram um estudo com o objetivo de conhecer as percepções de saúde bucal dos indivíduos com 60 anos ou mais que utilizam medicamentos anti-hipertensivos e participam do Programa de Saúde da Família (PSF) no município de Governador Valadares (Minas Gerais). Participaram do estudo 59 indivíduos, portadores de Hipertensão Arterial Sistêmica (HAS), de ambos os sexos, de estado funcional independente ou parcialmente dependente, não diabéticos e não tabagistas. Foi feita uma entrevista semiestruturada visando obter dados quanto à percepção e aos sentimentos desses indivíduos em relação à sua saúde bucal. Os dados foram analisados utilizando-se a técnica da Análise de Conteúdo. Os resultados revelaram que a maioria dos idosos ressaltou a importância dos dentes e das próteses, a necessidade de cuidados diários e tratamento dentário, concluindo que os idosos demonstraram uma percepção mais positiva que negativa sobre seus dentes, apesar de os problemas bucais terem impacto negativo nas suas vidas.

Rezende ${ }^{15}$ buscou, em seu estudo, estabelecer um perfil da percepção e realização dos cuidados de saúde bucal prestados aos pacientes idosos durante suas internações hospitalares pelo corpo de enfermagem. Realizou 209 entrevistas com profissionais da equipe de enfermagem que atuam em instituições hospitalares públicas e privadas, prestando atendimento à pacientes idosos na cidade de Uberlândia (MG). Os resultados sugerem que os cuidados de higiene oral realizados nos pacientes idosos hospitalizados são escassos e inadequados, tornando-se necessárias modificações nos cuidados dispensados, atualmente, no ambiente hospitalar pela equipe de enfermagem. A presença de um cirurgião-dentista, a difusão dos conhecimentos de odontologia preventiva e o uso de recursos específicos de higiene oral são medidas sugeridas como tentativas de solucionar as dificuldades apresentadas na manutenção da saúde oral e no tratamento das doenças bucais, que afetam a saúde geral dos indivíduos idosos hospitalizados. A atuação interdisciplinar no atendimento geriátrico é defendida visando obter melhor qualidade de vida dos idosos.

Alcântara et al. ${ }^{16}$ compararam as condições de saúde bucal de 618 idosos, institucionalizados e não institucionalizados, residentes no município de Governador Valadares (MG). Os dados foram coletados no período de dezembro de 2007 a março de 2008. As variáveis incluídas para identificar a saúde bucal foram CPOD (índice de dentes cariados, perdidos e obturados), uso e necessidade de prótese dentária. A média de idade foi de 76,1 para os institucionalizados e de 69,9 para os não institucionalizados. Em relação ao gênero há uma diferença significativa entre os grupos, predominando o feminino. Quanto ao estado civil, nos institucionalizados prevalecem os homens solteiros $(93,1 \%)$ e, nos não institucionalizados, as mulheres casadas (76,9\%). O CPOD médio dos idosos institucionalizados foi 31,6, não existindo diferença significativa desta variável no tocante ao gênero. Nos idosos não institucionalizados o CPOD médio foi 24,52, prevalecendo a semelhança entre os sexos. O tipo de prótese removível mais utilizada pelos idosos foi a total superior $(77,2 \%)$. Constata-se que $80,8 \%$ dos idosos institucionalizados não utilizam nenhum tipo de prótese, enquanto para os não institucionalizados este índice é de 43,6\%. Idosas não institucionalizadas $(40,2 \%)$ apresentam menor necessidade de prótese que as institucionalizadas. Verifica-se que a condição de saúde bucal de idosos residentes em instituições de longa permanência tende a ser inferior àquela de idosos não institucionalizados devido à alta prevalência de cárie e edentulismo revelada pelo índice CPOD. Supõe-se que tal ocorrência se deve à falta de recursos econômicos (pessoal insuficiente para cuidados de higiene bucal dos idosos), à dificuldade de acesso dos idosos aos serviços odontológicos e à ausência de um cirurgião-dentista na equipe, dificultando o acompanhamento e o treinamento dos cuidadores para o exercício das atividades de higiene bucal.

Ferreira et al. ${ }^{17}$ avaliaram a atuação de cirurgiões-dentistas nas instituições de longa permanência de Belo Horizonte e as práticas de higiene bucal adotadas. Questionários semiestruturados foram entregues aos coordenadores das 37 instituições filantrópicas e 30 privadas e coletados após uma semana. Houve retorno de $81 \%$ dos questionários. A maioria das instituições priva- 
das $(74,2 \%)$ e filantrópicas ( $87 \%$ ) não possui CD na equipe de saúde $(\mathrm{p}=0,21)$. A localização da instituição, o tempo de fundação, o tipo e o número de residentes não interferiram na presença de $C D(p>0,05)$. Nas instituições filantrópicas com consultório, $67 \%$ possuíam CD e nenhum $\mathrm{CD}$ trabalhava onde não havia consultório. Mesmo sem consultório, $13 \%$ das privadas possuíam CD. 69,6\% das filantrópicas encaminhavam o idoso em caso de necessidade para centros de saúde e nas privadas, $58,1 \%$ direcionavam ao familiar $(\mathrm{p}=0,00)$. Maior percentual de privadas adotava medidas sistematizadas de higiene bucal $(\mathrm{p}=0,01)$, com grande variabilidade nas condutas relatadas. Assim, os autores concluíram que é real a necessidade de se incorporar o cirurgiãodentista na equipe de saúde das instituições e de sistematizar as práticas de higiene bucal.

Silva e Fernandes ${ }^{18}$ avaliaram a percepção da condição bucal em idosos pela aplicação de um questionário com perguntas voltadas para a autoavaliação da situação bucal, e analisaram os fatores clínicos, subjetivos e sociodemográficos que interferem nessa percepção. Participaram do estudo 201 pessoas, dentadas, com 60 anos ou mais, funcionalmente independentes, que frequentavam um centro de saúde localizado em Araraquara (SP). Os pesquisadores realizaram também um exame clínico para determinar a prevalência das principais doenças bucais. O exame clínico revelou grande prevalência das principais doenças bucais, apesar de $42,7 \%$ das pessoas avaliarem sua condição bucal como regular. As variáveis associadas à autoavaliação foram: classe social, índice de GOHAI, dentes cariados e indicadores de extração. A análise multivariada mostrou que os preditores da autoavaliação foram o GOHAI, os dentes com extração indicada e o índice Community Periodontal Index and Treatment Needs, sendo que esses preditores explicaram 30\% da variabilidade da autoavaliação. Os autores concluíram assim que a percepção da saúde bucal teve pouca influência na condição clínica, indicando ser necessário o desenvolvimento de ações preventivas e educativas para a população.

Veras e Caldas ${ }^{19}$, em seu estudo, mencionam o movimento Universidades da Terceira Idade, que vem experimentando incremento substancial desde os anos 70, difundindo conceitos e experiências práticas que representam uma nova forma de promover a saúde da pessoa que envelhece, a partir de uma ação interdisciplinar comprometida com a inserção do idoso como cidadão ativo na sociedade. O movimento visa contribuir para a promoção da saúde física, mental e social das pessoas idosas, lançando mão das possibilidades existentes nas universidades. No Brasil, existem pelo menos 150 programas dessa natureza. Os resultados vêm sendo sistematizados e debatidos, trazendo novas perspectivas de inserção e ampliação da participação social e de melhoria das condições de saúde e qualidade de vida dos seus participantes. O artigo discute a importância do movimento como estratégia para a melhoria da qualidade de vida da população idosa. A partir da contextualização do movimento no mundo e no Brasil, uma experiência desenvolvida na Universidade do Estado do Rio de Janeiro (Uerj), desde 1993, vem sendo descrita e analisada. Tendo como pressupostos básicos a interdisciplinaridade, a participação social e a promoção da saúde, a proposta da Uerj utiliza metodologia adaptada às especificidades dessa clientela no desenho de um programa amplo de atenção integral à saúde do idoso.

Para Pucca Júnior ${ }^{4}$, os recursos básicos para orientação do idoso envolvem informações quanto à limpeza regular diária dos dentes e próteses, quanto ao controle da dieta e orientações visando ao fortalecimento da superfície dentária, com o uso do flúor.

Segundo Varellis ${ }^{6}$, para controle da cárie e da doença periodontal são usadas medidas preventivas, como controle da placa bacteriana, avaliação e estimulação da função mastigatória, fluorterapia, estimuladores de saliva e aconselhamento dietético, entre outras.

Os métodos preventivos não são apenas imprescindíveis como também se constituem como eixo de qualquer intervenção que vise à saúde bucal na terceira idade ${ }^{4}$.

\section{Considerações finais}

Tanto as fontes revisadas quanto a literatura que serviu de base para a realização deste estudo apontam para uma questão fundamental: a população idosa é o segmento populacional que mais cresce. Assim, a profissão odontológica, incluindo suas associações, deve estar ciente e alerta para essa questão, de forma a ampliar o estudo e a pesquisa nessa área, visando qualificar a atenção e o tratamento odontológico para esses indivíduos.

A saúde bucal na terceira idade é um fator indispensável para o envelhecimento saudável e uma boa qualidade de vida. Entretanto, as condições desiguais em que as pessoas vivem e trabalham são refletidas nitidamente na saúde bucal, uma vez que idosos expostos a situações de 
vulnerabilidade social estão mais sujeitos à interferência direta dos determinantes sociais no processo saúde-doença.

Os problemas bucais mais prevalentes nessa faixa etária são as cáries radiculares e a doença periodontal, que contribuem para a grande maioria das extrações dentárias. Embora o edentulismo seja considerado por muitos um fenômeno natural do envelhecimento, podemos entender que ele é o reflexo da falta de orientação e de cuidados com a saúde bucal em todas as idades.

Verificamos que na Região Sudeste do Brasil o uso de prótese é baixo quando comparado à alta taxa de edentulismo e ao percentual de idosos parcialmente dentados. Apresentou um alto valor no índice CPOD. Os idosos apresentam uma percepção positiva sobre seus dentes, apesar de os problemas bucais terem impacto nega- tivo nas suas vidas. A incorporação do cirurgiãodentista na equipe de saúde das instituições e a sistematização das práticas de higiene bucal são necessidades reais.

A estratégia para mudar essa realidade que prevalece atualmente é trabalhar preventivamente de forma que as pessoas possam chegar à idade mais avançada com uma dentição ainda saudável. E para aqueles que já perderam seus dentes, deve-se assegurar seu acesso à reabilitação das funções mastigatórias e estética, através da confecção de próteses dentárias. Além disso, faz-se necessário um trabalho continuado de orientação para o cuidado com a sua própria saúde bucal, incluindo desde instruções para a limpeza diária dos dentes e próteses até o controle da dieta e o uso do flúor como fonte de fortalecimento da superfície dentária.

\section{Colaboradores}

ACA Simões trabalhou na concepção da proposta, na coleta e análise dos dados, na conclusão e redação do artigo; DM Carvalho, na concepção, metodologia e revisão final. 


\section{Referências}

1. Cormack EF. A saúde oral do idoso. [acessado 2006 set 10]. Disponível em: http://www.odontologia. com.br/artigos/geriatria.html

2. Silva AL, Saintrain MVL. Interferência do perfil epidemiológico do idoso na atenção odontológica. Rev Bras Epidemiologia 2006; 9(2):242-250.

3. Brunetti RF, Galo R, Vitti M, Santos CM, Hallak JEC, Regalo SCH. Odontogeriatria: noções de interesse clínico. São Paulo: Artes Médicas; 2002.

4. Pucca Júnior GA. Saúde bucal do idoso: aspectos sociais e preventivos. In: Papaleo NM, organizador. Gerontologia: a velhice e o envelhecimento em versão globalizada. São Paulo: Atheneu; 2002. p. 297310.

5. Colussi CF, Freitas SFT. Aspectos epidemiológicos da saúde bucal do idoso no Brasil. Cad Saude Publica 2002; 18(5):1313-1320.

6. Varellis MLZ. O paciente com necessidades especiais na odontologia. São Paulo: Santos; 2005.

7. Fiaminghi DL, Dummel J, Padilha DMP, Moro RGD. Odontogeriatria: a importância da autoestima na qualidade de vida do idoso. Rev Clín Pesq Odontol 2004; 1(2):37-40.

8. Araújo SSC, Freire DBL, Padilha DMP, Baldisserotto J. Suporte social, promoção de saúde e saúde bucal na população idosa no Brasil. Interface - Comunic, Saúde, Educ 2006; 10(19):203-216.

9. Carneiro RMV, Silva DD, Sousa MLR, Wada RS. Saúde bucal de idosos institucionalizados, zona leste de São Paulo, Brasil, 1999. Cad Saude Publica 2005; 21(6):1709-1716.

10. Meneghim MC, Pereira AC, Silva FRB. Prevalência de cárie radicular e condição periodontal em uma população idosa institucionalizada de PiracicabaSP. Pesqui Odontol Bras 2002; 16(1):50-56.

11. Silva SRC, Valsecki Júnior A. Avaliação das condições de saúde bucal dos idosos em um município brasileiro. Rev Panam Salud Publica 2000; 8(4):268271.

12. Moreira RS, Nico LS, Tomita NE, Ruiz T. A saúde bucal do idoso brasileiro: revisão sistemática sobre o quadro epidemiológico e acesso aos serviços de saúde bucal. Cad Saude Publica 2005; 21(6):16651675.
13. Ferreira RC, Magalhães CS, Rocha ES, Schwambach CW, Moreira AN. Saúde bucal de idosos residentes em instituições de longa permanência de Belo Horizonte [tese]. Belo Horizonte: Universidade Federal de Minas Gerais; 2007.

14. Rodrigues SM, Vargas AMD, Moreira AN. Percepção de saúde bucal em idosos. Arq Odontol 2003; 39(3):195-212.

15. Rezende TO. Análise dos cuidados bucais realizados pela enfermagem nos idosos hospitalizados em Uberlândia. São Paulo: Abeno; 2005.

16. Alcântara CM, Freitas CA, Dias CA, Gonçalves C, Dias LCS, Rodrigues SM, Pereira VG. Estudo comparativo da condição de saúde bucal de idosos institucionalizados e não institucionalizados de Governador Valadares. Painel apresentado $1^{\circ}$ Congresso Nacional de Saúde da Faculdade de Medicina da UFMG; 2008; Minas Gerais.

17. Ferreira RC, Schwambach CW, Magalhães CS, Moreira AN. Atenção odontológica e práticas de higiene bucal em instituições de longa permanência geriátricas. [documento da Internet]. [acessado $2008 \mathrm{dez}$ 3]. Disponível em: http://www.abrasco.org.br/ cienciaesaudecoletiva/artigos/index.php

18. Silva SRC, Fernandes, RAC. Autopercepção das condições de saúde bucal por idosos. Rev Saude Publica 2001; 35(4):349-355.

19. Veras RP, Caldas CP. Promovendo a saúde e a cidadania do idoso: o movimento das universidades da terceira idade. Cien Saude Colet 2004; 9(2):423-432.

Artigo apresentado em 21/07/2008

Aprovado em 26/12/2008

Versão final apresentada em 10/01/2009 